\title{
THE CLINICAL TRIAL OF EIGHTEEN ANALOGUES OF PAMAQUIN (PLASMOCHIN) IN VIVAX MALARIA (CHESSON STRAIN) ${ }^{1}$
}

\author{
By ALF S. ALVING, THEODORE N. PULLMAN,2 BRANCH CRAIGE, JR.,2 RALPH \\ JONES, JR.,2 C. MERRILL WHORTON,2 AND LILLIAN EICHELBERGER
}

(From the Malarial Research Unit, Department of Medicine, University of Chicago)

(Received for publication February 14, 1947)

In the malarial research program conducted on a national scale during World War II, several drugs were developed which had marked superiority over quinacrine (atabrine) and quinine in their ability to terminate individual attacks of vivax malaria (1). For example, chloroquine $(\mathrm{SN}-7618)$ in terms of oral dosage and the resulting plasma concentration can be administered in amounts many times that required to suppress the disease (2). The margin between the therapeutic and toxic dose of chloroquine is several times that which exists for quinine and quinacrine. When these new compounds, however, failed, even at high dosages, to affect the relapse rate it seemed doubtful that further extension of research aimed chiefly towards finding a more effective and less toxic, and primarily suppressive, drug would lead

\footnotetext{
1 This investigation was carried out under contract, recommended by the Committee on Medical Research, between the Office of Scientific Research and Development and the University of Chicago. The studies were planned in cooperation with the Panel on Clinical Testing of Antimalarials of the Board for the Coordination of Malarial Studies. This work was further aided by the participation of Army Medical officers assigned to the project by the Surgeon General, U. S. Army.

Through a cooperative arrangement between Professor Clay G. Huff and Dr. Frederick Coulston, Department of Bacteriology and Parasitology, and the Malarial Research Unit, Department of Medicine, the former group bred Anopheles quadrimaculatus mosquitoes, supervised their infection and the inoculation of volunteers, and determined the intensity of infection in the salivary glands of the mosquitoes. The latter group assumed the responsibility for clinical care of patients studied by both groups.

The authors express their thanks to the Malaria Study Section of the National Institute of Health for editorial assistance and for arrangements in regard to the publication of this paper. They are also grateful to the Abbott Laboratories, E. I. du Pont de Nemours and Company, Inc., E. R. Squibb and Sons, Eli Lilly and Company, Sharp and Dohme, Inc., and Wyeth, Inc., for contributing toward the publication costs.

2 Formerly Captain, M.C., A.U.S.
}

to early development of a compound that would effect a radical cure.

Pamaquin (plasmochin) has been recognized for many years as an antimalarial drug with unusual properties ( 3 to 9 ). It has proved more efficacious in reducing the relapse rate of vivax malaria than previously used drugs. Because of its toxicity, however, pamaquin fell into disrepute and its use in the Armed Services of the United States was discontinued (10).

The most direct approach to the problem was to explore the chemical analogues of pamaquin in the hope of developing a compound which possessed the therapeutic properties of pamaquin but which was free of its undesirable toxic effects. Consequently, in 1944, the national program was realigned, the major emphasis being placed on pamaquin analogues. The chemical structure of pamaquin is shown in Figure 1. It is a member

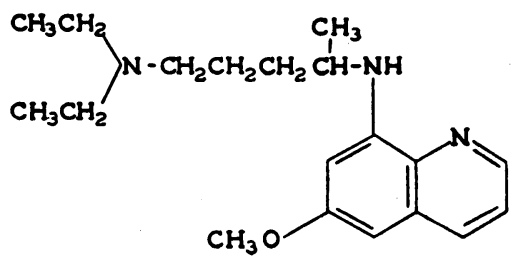

Fig. 1. Structural Formula of Pamaquin (Plasmochin)

of a class of compounds in which there is a substituted aminoalkyl group on an 8-amino radical on a quinoline nucleus (Figure in Table I). Pamaquin and 21 other substituted 8-aminoquinolines have been studied clinically in human volunteers.

\section{METHODS AND PROCEDURES}

General. Details of the general procedure and the plan of ubservation are reported elsewhere (11). Healthy, presumably susceptible white volunteers at Stateville 


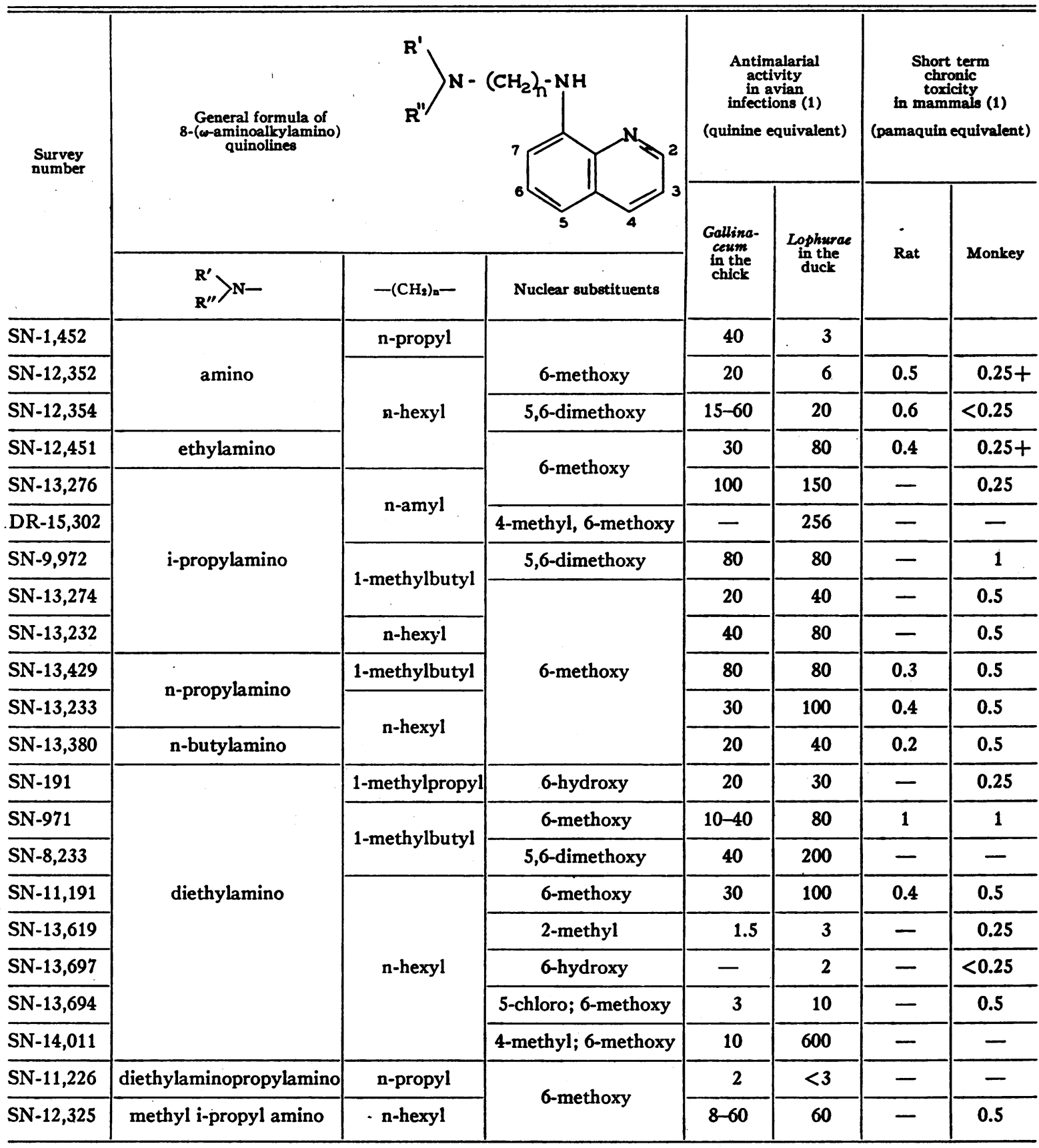

Penitentiary, ${ }^{3}$ which is located in a non-endemic area, were infected with Southwest Pacific vivar malaria (Chesson strain) (12) by the bites of infected Anopheles

3 The investigations reported in this paper would not have been possible except for the enthusiastic cooperation of the inmates and the administrative staff of Stateville Penitentiary. quadrimaculatus mosquitoes. This strain is characterized by a high relapse rate when treated with non-curative drugs such as quinine and quinacrine, by a short period of latency between successive attacks, and by almost complete absence of delayed primary attacks $(13,14)$. Volunteers undergoing primary attacks and first or second relapses served as test subjects. Treatment was initiated promptly after the appearance of fever and para- 
sitemia in order to minimize the effects of acquired immunity. Five volunteers composed a test group in the standard procedure. When a compound showed promise, however, studies were expanded to larger groups and different dosages.

Selection of drugs. Only pamaquin analogues with pamaquin-type toxicity in animals were used; no plasmacid type compounds received trial in man. As new drugs were synthesized, they were tested in avian infections for antimalarial activity and in rodents, dogs, and monkeys for toxicity. On the basis of the animal experiments, the drugs that offered promise received clinical testing in man. Table I summarizes the chemical structure, avian antimalarial activity and mammalian toxicity of the drugs reported in this paper. They were all 8-( $\omega$-aminoalkylamino) quinolines, differing from each other in nuclear substituents, length and character of the inter-nitrogen aliphatic chain, and the number and nature of substituents on the terminal amine nitrogen.

Dosage. Early in the course of the drug-testing program it had been planned to conduct the human clinical trials at approximately the maximum tolerated dose as estimated from the animal experiments. However, severe toxic reactions resulted from the use of four of these drugs ${ }^{4}$ at this high dosage and this approach was abandoned. Pamaquin is therapeutically efficacious at a daily dose of $90 \mathrm{mgm}$. of base (equivalent to $200 \mathrm{mgm}$. of the naphthoate) and is well tolerated at a dose of 15 mgm. of base per day. Since a new drug, to be ideal, should possess at least the therapeutic efficacy of pamaqin at $90 \mathrm{mgm}$. (base) and no greater toxicity than that of pamaquin at $15 \mathrm{mgm}$. (base), it was decided to test new drugs at $15 / 90$, or $1 / 6$, of their maximum tolerated dose as estimated from monkey experiments. If, at this dosage, human toxicity was found to be absent or mild, the drugs were tested at a higher dosage level.

Administration of drugs. The drugs were administered over a 14-day period in equal doses every four hours to insure fairly constant concentrations in the body fluids. Quinine was administered concurrently with the drugs for two reasons: (1) the synergistic effect of quinine on pamaquin may also extend to pamaquin analogues, and (2) if it is assumed that a curative agent may prevent relapse by action chiefly on hypothetical exo-erythrocytic stages of the parasite with little or no action on the erythrocytic stages, the concurrent administration of an anti-trophozoite agent is indicated. Qui-

$4 \mathrm{SN}-8,233, \mathrm{SN}-11,191$, and $\mathrm{SN}-11,226$ were tested at the maximum tolerated dose as estimated from monkey toxicity. SN-1,452 was tested at half the estimated maximum tolerated dose. Agranulocytosis ( $\mathrm{SN}-8,233$ ), granulocytopenia (SN-11,191 and SN-11,226), drug fever $(\mathrm{SN}-1,452)$ and high methemoglobinemia ( $\mathrm{SN}-8,233$ and $\mathrm{SN}-11,191)$ were encountered. One of three subjects to whom SN-8,233 was administered died of myocardial infarction nine days after drug was stopped. Death was thought to be unrelated to drug toxicity (1). Incidentally, no unequivocal therapeutic effect was obtained. nine was given as the dihydrochloride or sulfate in doses of 2.0 grams of the salt daily, in six equally divided doses at four-hour intervals.

Classification of cases. It has been shown elsewhere (14) that under the standardized conditions of this investigation, the relapse rate of the Chesson strain is high when suppressive drugs are used. Some individuals, however, fail to relapse although treated with drug regimes ordinarily considered to possess only suppressive activity. It has been possible to relate probability of relapse with the length of the prepatent and latent periods (14). The relapse rate after treatment of attacks in patients who had prepatent periods less than 15 days or latent intervals less than 30 days was 98 per cent, whereas it was only 67 per cent when the prepatent or latent periods were longer. On the basis of length of prepatent and latent periods, therefore, this series of cases has been divided into two groups. One group consists of individuals whose prepatent periods were 14 days or less and whose preceding latent intervals were 29 days or less. This group offers a more severe therapeutic test for an antimalarial agent than the remainder of the cases. The latter group, with longer prepatent and latent periods, offers only a moderate therapeutic test for an antimalarial drug, because of lower probability of relapse.

Chemical estimation of drugs in the plasma. Blood was drawn for analysis every other day during the 14day course of treatment and for four days thereafter.

Oxalated whole blood was centrifuged for 15 minutes at 2,000 r.p.m., the plasma was separated and re-centrifuged for 60 minutes at the same speed to insure complete removal of the components of the buffy coat.

Quinine was estimated by the method of Brodie and Udenfriend (15). The 8-aminoquinolines were estimated by the method of Brodie, Udenfriend and Taggart (16) modified as follows: $20 \mathrm{ml}$. of heptane and $0.5 \mathrm{ml}$. of iso-butyl alcohol were placed in a $60-\mathrm{ml}$. glass stoppered bottle. Ten ml. of plasma and $10 \mathrm{ml}$. of $0.1 \mathrm{~N} \mathrm{NaOH}$ were added and the mixture shaken for 10 minutes. The mixture was then transferred to a $50-\mathrm{ml}$. centrifuge tube and centrifuged for five minutes. The water phase was aspirated and $15 \mathrm{ml}$. of the heptane phase was transferred to a $40-\mathrm{ml}$. glass-stoppered pointed centrifuge tube containing $0.5 \mathrm{ml}$. of coupling reagent (diazotized sulphanilic acid). The mixture was then shaken for five minutes and centrifuged. The heptane layer was removed by aspiration. Not less than $0.3 \mathrm{ml}$. of the water layer was transferred to a special microcuvette and the transmission read in a spectrophotometer (Coleman) at a wave length of 480 millimicra.

Average plasma concentrations of quinine and test drug were arrived at by calculation of the arithmetic mean of the seven samples taken during treatment.

Hemoglobin and methemoglobin. Hemoglobin and methemoglobin determinations were performed daily on venous blood during treatment using the method of Wendell (17). 
TABLE II

Therapeutic effect in sporozoite-induced vivax malaria of eighteen 8-aminoquinolines administered for 14 days concurrently with quinine

\begin{tabular}{|c|c|c|c|c|c|c|c|c|c|c|c|c|}
\hline \multirow{3}{*}{ Drug } & \multirow{3}{*}{$\begin{array}{l}\text { Daily } \\
\text { dose } \\
\text { (base) }\end{array}$} & \multirow{3}{*}{$\begin{array}{l}\text { Num- } \\
\text { ber } \\
\text { of } \\
\text { sub- } \\
\text { jects }\end{array}$} & \multicolumn{4}{|c|}{$\begin{array}{l}\text { Mean concentration of } \\
\text { drug in plasma }\end{array}$} & \multicolumn{2}{|c|}{$\begin{array}{c}\text { Relapse ratio } \\
\text { Individuals relapsed } \\
\text { Individuals treated }\end{array}$} & \multicolumn{2}{|c|}{$\begin{array}{l}\text { Duration of subse- } \\
\text { quent parasitic } \\
\text { latent period in } \\
\text { subjects who relapsed }\end{array}$} & \multicolumn{2}{|c|}{$\begin{array}{l}\text { Duration of follow-up } \\
\text { period in subjects } \\
\text { who did not } \\
\text { relapse }\end{array}$} \\
\hline & & & \multicolumn{2}{|c|}{ Test drug } & \multicolumn{2}{|c|}{ Quinine } & \multirow{2}{*}{$\begin{array}{l}\text { Mod- } \\
\text { erate* } \\
\text { infec- } \\
\text { tions }\end{array}$} & \multirow{2}{*}{$\begin{array}{c}\text { Severe* } \\
\text { infec- } \\
\text { tions }\end{array}$} & \multirow{2}{*}{$\begin{array}{c}\text { Mod- } \\
\text { erate* } \\
\text { infec- } \\
\text { tions }\end{array}$} & \multirow{2}{*}{$\begin{array}{c}\text { Severe* } \\
\text { infections }\end{array}$} & \multirow{2}{*}{$\begin{array}{l}\text { Moderate* } \\
\text { infections }\end{array}$} & \multirow{2}{*}{$\begin{array}{c}\text { Severe** } \\
\text { infections }\end{array}$} \\
\hline & & & Range & Mean & Range & Mean & & & & & & \\
\hline SN-191 & $\begin{array}{l}m g m . \\
64\end{array}$ & 5 & $\begin{array}{c}\text { gamma } \\
\text { per } \\
\text { liver } \\
\text { no } \\
\text { method }\end{array}$ & $\begin{array}{c}\text { gamma } \\
\text { per } \\
\text { liker }\end{array}$ & $\begin{array}{c}\text { mgm. } \\
\text { per } \\
\text { liter } \\
5-6\end{array}$ & $\begin{array}{c}\text { mgm. } \\
\text { per } \\
\text { liter } \\
5 \\
5\end{array}$ & $1 / 1$ & $4 / 4$ & $\begin{array}{l}\text { days } \\
13\end{array}$ & $\begin{array}{c}\text { days } \\
9,11,18,13\end{array}$ & days & days \\
\hline \multirow[b]{2}{*}{ SN-9,972 } & 7.5 & 5 & $\begin{array}{c}\text { no } \\
\text { method }\end{array}$ & - & $7-8$ & 7 & - & $3 / 5$ & & $12,16,62$ & & 124,137 \\
\hline & 15 & 12 & $\begin{array}{c}\text { no } \\
\text { method }\end{array}$ & - & $5-10$ & 8 & $0 / 2$ & $2 / 10$ & & 78,11 & 214,164 & $\begin{array}{l}122,118, \\
114,117, \\
118,112, \\
81,81\end{array}$ \\
\hline SN-11,191 & 30 & 5 & $92-232$ & 143 & $6-11$ & 9 & - & $5 / 5$ & & $\begin{array}{c}29,37,26 \\
11,16\end{array}$ & & \\
\hline \multirow{2}{*}{ SN-12,325 } & 30 & 5 & $80-103$ & 94 & $5-10$ & 8 & $0 / 3$ & $2 / 2$ & & 14,12 & $304,301,296$ & \\
\hline & 60 & 5 & |166-307 & 247 & $6-11$ & 9 & $2 / 3$ & $1 / 2$ & 52,33 & 106 & 216 & 213 \\
\hline \multirow{2}{*}{ SN-12,352 } & 30 & 5 & $0-25$ & 12 & $6-10$ & 8 & $1 / 2$ & $3 / 3$ & 12 & $10,8,20$ & 268 & \\
\hline & 60 & 5 & $27-43$ & 35 & $6-9$ & 7 & $2 / 3$ & $1 / 2$ & 12,44 & 19 & 227 & 227 \\
\hline \multirow{2}{*}{ SN-12,354 } & 30 & 2 & $6-9$ & 8 & $7-10$ & 8 & - & $2 / 2$ & & 18,35 & & \\
\hline & 60 & 5 & $10-31$ & 15 & $7-9$ & 8 & $1 / 3$ & $2 / 2$ & 26 & 14,10 & 328,262 & \\
\hline \multirow{2}{*}{ SN-12,451 } & 30 & 3 & $59-84$ & 75 & $9-10$ & 10 & $0 / 1$ & $2 / 2$ & & 28,10 & 281 & \\
\hline & 60 & 5 & $83-216$ & 147 & $6-10$ & 8 & $0 / 1$ & $3 / 4$ & & $12,49,12$ & 245 & 250 \\
\hline \multirow{2}{*}{ SN-13,232 } & 30 & 5 & $76-123$ & 92 & 5-11 & 8 & $0 / 2$ & $3 / 3$ & & $28,8,13$ & 352,302 & \\
\hline & 60 & 5 & $185-291$ & 211 & $8-11$ & 9 & $1 / 2$ & $3 / 3$ & 53 & $19,23,49$ & 236 & \\
\hline SN-13,233 & 60 & 5 & $65-156$ & 114 & $8-11$ & 9 & $0 / 1$. & $3 / 4$ & & $16,10,10$ & 266 & 280 \\
\hline \multirow{2}{*}{ SN-13,274 } & 30 & 5 & $24-41$ & 32 & $5-11$ & 8 & $1 / 1$ & $2 / 4$ & 15 & 13,19 & & 222,222 \\
\hline & 60 & 10 & $26-285$ & 70 & $6-9$ & 7 & $0 / 5$ & $1 / 5$ & & 23 & $\begin{array}{c}157,167,158 \\
152,107\end{array}$ & $\begin{array}{l}83,83 \\
83,80\end{array}$ \\
\hline \multirow{4}{*}{ SN-13,276 } & 15 & 5 & $30-51$ & 40 & $7-11$ & 9 & - & $5 / 5$ & & $\begin{array}{c}21,15,37 \\
12,9\end{array}$ & & \\
\hline & 30 & 10 & $33-67$ & 44 & $5-10$ & 8 & $0 / 1$ & $8 / 9$ & & $\begin{array}{l}41,41,17 \\
48,11,38 \\
64,12\end{array}$ & 187 & 192 \\
\hline & 45 & 5 & $24-58$ & 43 & $5-10$ & 8 & $0 / 3$ & $2 / 2$ & & 15,14 & $208,183,181$ & \\
\hline & 60 & 43 & 26-196 & 63 & $3-11$ & 7 & $1 / 26$ & $3 / 17$ & 22 & $70,45,54$ & $\begin{array}{l}188,179,177, \\
166,164,165, \\
164,143,158, \\
149,131,130 \\
124,149,122, \\
116,111,108, \\
105,76,73, \\
73,65,63,80\end{array}$ & \begin{tabular}{|l}
370,380, \\
352,349, \\
327,271 \\
270,270, \\
267,124, \\
81,81, \\
61,65
\end{tabular} \\
\hline
\end{tabular}




\begin{tabular}{|c|c|c|c|c|c|c|c|c|c|c|c|c|}
\hline \multirow{3}{*}{ Drug } & \multirow{3}{*}{$\begin{array}{l}\text { Daily } \\
\text { dose } \\
\text { (base) }\end{array}$} & \multirow{3}{*}{$\begin{array}{l}\text { Num- } \\
\text { ber } \\
\text { of } \\
\text { sub- } \\
\text { jects }\end{array}$} & \multicolumn{4}{|c|}{$\begin{array}{l}\text { Mean concentration of } \\
\text { drug in plasma }\end{array}$} & \multicolumn{2}{|c|}{$\begin{array}{c}\text { Relapse ratio } \\
\text { Individuals relapsed } \\
\text { Individuals treated }\end{array}$} & \multicolumn{2}{|c|}{$\begin{array}{l}\text { Duration of subse- } \\
\text { quent parasitic } \\
\text { latent period in } \\
\text { subjects who relapsed }\end{array}$} & \multicolumn{2}{|c|}{$\begin{array}{l}\text { Duration of follow-up } \\
\text { period in subjects } \\
\text { who did not } \\
\text { relapse }\end{array}$} \\
\hline & & & \multicolumn{2}{|c|}{ Test drug } & \multicolumn{2}{|c|}{ Quinine } & \multirow{2}{*}{$\begin{array}{l}\text { Mod- } \\
\text { erate* } \\
\text { infec- } \\
\text { tions }\end{array}$} & \multirow{2}{*}{$\begin{array}{c}\text { Severe* } \\
\text { infec- } \\
\text { tions }\end{array}$} & \multirow{2}{*}{$\begin{array}{l}\text { Mod- } \\
\text { erate* } \\
\text { infec- } \\
\text { tions }\end{array}$} & \multirow{2}{*}{$\begin{array}{c}\text { Severe* } \\
\text { infections }\end{array}$} & \multirow{2}{*}{$\begin{array}{c}\text { Moderate* } \\
\text { infections }\end{array}$} & \multirow{2}{*}{$\begin{array}{c}\text { Severe* } \\
\text { infections }\end{array}$} \\
\hline & & & Range & Mean & Range & Mean & & & & & & \\
\hline SN-13,380 & $\begin{array}{l}m g m . \\
60\end{array}$ & 5 & $\begin{array}{c}\text { gamma } \\
\text { per } \\
\text { liter } \\
99-251\end{array}$ & $\begin{array}{l}\text { gamma } \\
\text { pert } \\
\text { liter } \\
152\end{array}$ & $\begin{array}{l}\text { mgm. } \\
\text { per } \\
\text { liter } \\
6-12\end{array}$ & $\begin{array}{c}\text { mgm. } \\
\text { per } \\
\text { liter } \\
9\end{array}$ & $1 / 2$ & $3 / 3$ & $\begin{array}{c}\text { days } \\
78\end{array}$ & $\begin{array}{l}\text { days } \\
16,13,12\end{array}$ & $\begin{array}{l}\text { days } \\
282\end{array}$ & days \\
\hline \multirow{2}{*}{ SN-13,429 } & 30 & 5 & $22-37$ & 32 & $7-10$ & 9 & $0 / 1$ & $4 / 4$ & & $\begin{array}{c}39,11,40, \\
10\end{array}$ & 276 & \\
\hline & 60 & 5 & $37-128$ & 51 & $8-10$ & 9 & - & $2 / 5$ & & 104,63 & & $\begin{array}{c}120,120 \\
120\end{array}$ \\
\hline SN-13,619 & 60 & 5 & $197-328$ & 242 & $8-10$ & 7 & - & $5 / 5$ & & $\begin{array}{c}16,9,31 \\
13,10\end{array}$ & & \\
\hline \multirow{2}{*}{ SN-13,694 } & 30 & 5 & $\begin{array}{c}\text { no } \\
\text { method }\end{array}$ & - & $6-11$ & 8 & $1 / 1$ & $4 / 4$ & 31 & $9,28,11$ & & \\
\hline & 60 & 5 & $\begin{array}{c}\text { no } \\
\text { method }\end{array}$ & - & $6-11$ & 8 & - & $4 / 5$ & & $\begin{array}{c}24,12,10 \\
10\end{array}$ & & 126 \\
\hline SN-13,697 & 120 & 5 & $\begin{array}{c}\text { no } \\
\text { method }\end{array}$ & - & $7-10$ & 7 & $1 / 1$ & $4 / 4$ & 11 & $8,11,16$ & & \\
\hline \multirow{4}{*}{ SN-14,011 } & 3.7 & 5 & $\begin{array}{c}\text { no } \\
\text { method }\end{array}$ & - & $6-10$ & 8 & - & $5 / 5$ & & $8,11,9,8$ & & \\
\hline & 7.5 & 5 & $\begin{array}{c}\text { no } \\
\text { method }\end{array}$ & - & $5-10$ & 8 & - & $5 / 5$ & & $7,9,10,10$ & & \\
\hline & 15 & 5 & $\begin{array}{c}\text { no } \\
\text { method }\end{array}$ & - & $5-9$ & 8 & - & $5 / 5$ & & $\begin{array}{c}8,8,10,8 \\
14\end{array}$ & & \\
\hline & 30 & 5 & $\begin{array}{c}\text { no } \\
\text { method }\end{array}$ & - & $5-10$ & 8 & - & $5 / 5$ & & $\begin{array}{c}12,12,10 \\
9,23\end{array}$ & & \\
\hline \multirow{2}{*}{ DR-15,302 } & 30 & 5 & \multirow{2}{*}{$\begin{array}{c}\text { no } \\
\text { method }\end{array}$} & - & $5-9$ & 7 & - & $2 / 5$ & & 16,15 & & $96,89,84$ \\
\hline & 60 & 5 & & - & $5-10$ & 8 & - & $2 / 5$ & & 74,76 & & $93,91,91$ \\
\hline
\end{tabular}

"Infections were classified as "severe" when the prepatent period or preceding latent interval was less than 15 days or 30 days, respectively. When these periods were longer, the infections were classified as "moderate."

\section{RESULTS}

\section{Antimalarial activity}

Table II summarizes the data on the antimalarial activity of 18 compounds that we have studied systematically. Thirty-four drug regimes in 65 moderate and 165 severe therapeutic tests are tabulated.

Relapse rate. Eight of the drugs were ineffective at the dosages studied in clinical attacks that represented severe therapeutic tests. All the subjects treated with these drugs subsequently underwent relapse.

Ten compounds apparently cured one or more patients whose infection constituted a severe therapeutic challenge. Five of these drugs showed pronounced activity in that the majority of subjects treated with them have not subsequently relapsed. Follow-up observations on these patients have been made from two months to one year. This group of drugs consists of SN-9,972, SN$13,274, \mathrm{SN}-13,429$, and DR-15,302. SN-13,276 (pentaquine) has cured 14 out of 17 infections that fall into the severe test group. Studies on this drug have been expanded to include curative trials at different dosages with and without concurrent quinine, prophylactic tests, and more detailed studies on its toxicology in man $(18,19)$. 
Parasite clearance. The eighteen 8-aminoquinolines as a group, administered with quinine, terminated individual attacks no more rapidly than quinine alone. The 34 regimes were divided into two groups; the first consisted of those on which all relapsed, the second included those on which one or more individuals failed to relapse. The time required for clearance of parasites from the peripheral blood was then studied for all patients falling into each of the two groups. The difference between the mean parasite clearance times of the two groups was not statistically significant.

Latent period. The cumulative frequency of latent intervals in 110 subjects who relapsed after treatment with these drugs is shown in Figure 2. All subjects had infections classified as severe therapeutic tests. Fifty per cent of the cases relapsed within two weeks and 90 per cent within six weeks. These figures lend additional significance to the length of the follow-up periods noted in Table II.

The latent interval ogive may be broken down into three separate curves. The 34 regimes were divided into three groups according to the relapse ratio for therapeutic tests. The first group consisted of 73 attacks on 20 drug regimes on which all patients underwent subsequent relapse. The second group was comprised of 21 patients who relapsed on five drug regimes which produced a relapse rate of below 100 per cent but above 50

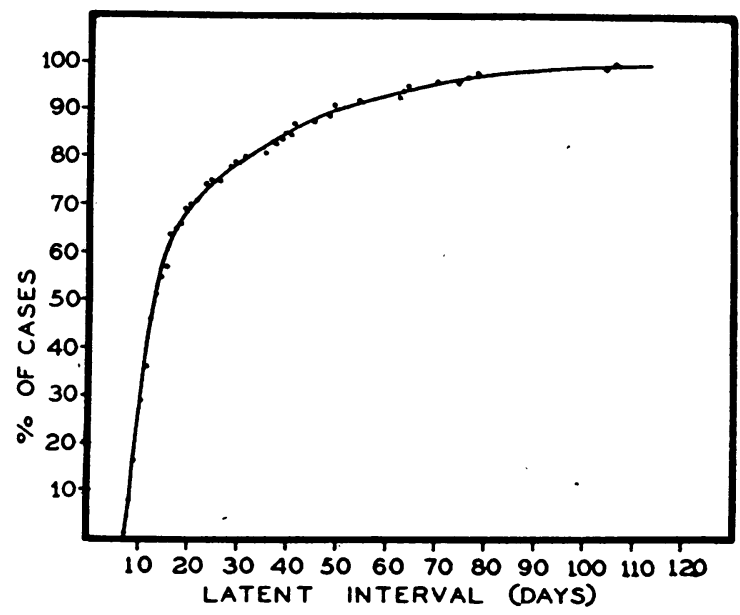

Fig. 2. Cumulative Frequency of Length of Latent Intervals in 110 Patients Who Relapsed after Treatment With 8-Aminoguinolines, AdMINISTEREd Concurrently With QUININE

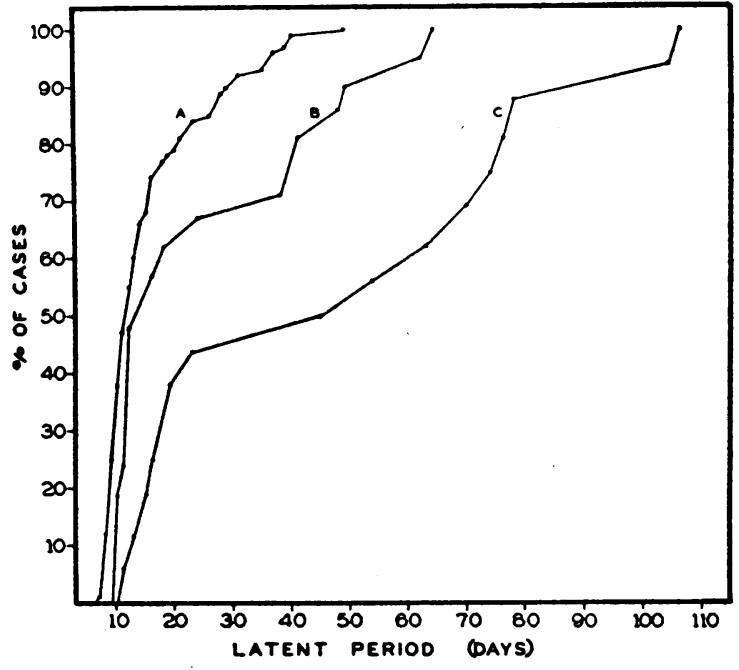

Fig. 3. Breakdown of Cumulative Frequency Ogive Shown IN Figure 2

Curve $A$ is based on the latent intervals of 73 patients on 20 drug regimes after which all patients relapsed. Curve $B$ is based on the latent intervals of 21 patients on five drug regimes after each of which more than onehalf of the patients relapsed. Curve $C$ is based on latent intervals of 16 patients on seven drug regimes after each of which half or less than half of the patients relapsed.

per cent. The third group was composed of 16 patients who relapsed on seven drug regimes on which 50 per cent or less of the patients relapsed. The curves are shown in Figure 3. It is apparent that drug regimes which produce a high percentage of cures, tend to prolong the subsequent latent intervals in patients whom they fail to cure.

Plasma concentrations. The plasma concentrations of the 8-aminoquinolines varied widely. In general, there was a very low positive correlation between oral dose and plasma concentrations but there were large individual variations. The concentration of the 8-aminoquinolines in the plasma could be correlated neither with the subsequent latent interval in those individuals who relapsed nor with relapse or failure to relapse. However, the total number of patients on any one drug regime was low.

The quinine plasma concentrations ranged between $3 \mathrm{mgm}$. per liter and $16 \mathrm{mgm}$. per liter in an approximately Gaussian distribution, with the mode occurring in the region of $8 \mathrm{mgm}$. per liter. There was no relation between the mean plasma quinine concentration attained during treatment 
and the length of the latent interval in those individuals who relapsed, nor was there any relation between quinine level and occurrence of relapse or failure to relapse.

When treatment was discontinued, the 8-aminoquinolines and quinine both disappeared rapidly from the plasma.

TOXICITY

Table III summarizes the data on the toxicity of the eighteen 8-aminoquinolines when given over a 14-day period concurrently with quinine.

\section{Symptomatology}

The symptoms produced by these regimes were qualitatively similar to those caused by pamaquin (9). At $15 \mathrm{mgm}$. of pamaquin daily, there were practically no symptoms, while at $63 \mathrm{mgm}$., abdominal pain, anorexia, nausea and vomiting were frequently severe and in several cases necessitated discontinuance of the medication before the 14 days of drug administration were completed. In Table III are indicated the clinical toxicities of each of the drug regimes tested and, as well, the

TABLE III

Toxicity in man of a group of eighteen 8-aminoquinolines administered for 14 days concurrently with quinine

\begin{tabular}{|c|c|c|c|c|c|c|c|c|c|c|}
\hline \multirow[t]{2}{*}{ Drug } & \multirow[t]{2}{*}{$\begin{array}{l}\text { Daily } \\
\text { dose } \\
\text { (base) }\end{array}$} & \multirow[t]{2}{*}{$\begin{array}{c}\text { Number } \\
\text { of } \\
\text { patients }\end{array}$} & \multicolumn{3}{|c|}{$\begin{array}{l}\text { Number of patients } \\
\text { showing commonly } \\
\text { observed symptoms }\end{array}$} & \multirow{2}{*}{$\begin{array}{l}\text { Number } \\
\text { of } \\
\text { patients } \\
\text { showing } \\
\text { leucocy- } \\
\text { tosis of } \\
12,000 \\
\text { or over }\end{array}$} & \multicolumn{2}{|c|}{$\begin{array}{l}\text { Average methemo- } \\
\text { globinemia* on } \\
\text { last } 4 \text { days of } \\
\text { treatment } \\
\text { per cent of total } \\
\text { hemoglobin }\end{array}$} & \multirow{2}{*}{$\begin{array}{c}\text { Approximate } \\
\text { pamaquin } \\
\text { (base) } \\
\text { equivalent } \\
\text { (concurrent } \\
\text { with } \\
\text { quinine) }\end{array}$} & \multirow[t]{2}{*}{ Comment } \\
\hline & & & Mild & $\begin{array}{l}\text { Mod- } \\
\text { erate }\end{array}$ & Severe & & Range & Mean & & \\
\hline SN-191 & $\begin{array}{l}m g m . \\
64\end{array}$ & 5 & 1 & 3 & 0 & 2 & $1.4-1.8$ & 1.6 & $\begin{array}{c}\text { mgm. per day } \\
30\end{array}$ & $\begin{array}{l}1 \text { patient had temperature } \\
\text { of } 100.6 \text { on } 10 \text { th day. }\end{array}$ \\
\hline \multirow[b]{2}{*}{ SN-9,972 } & $7 \frac{1}{2}$ & 5 & 1 & 1 & 0 & 1 & $1.8-3.1$ & 2.2 & 15 & \\
\hline & 15 & 12 & 3 & 4 & 0 & 4 & $1.9-5.9$ & 3.9 & 30 & $\begin{array}{l}1 \text { patient had white count } \\
\text { of } 3,700 \text { on } 14 \text { th day. }\end{array}$ \\
\hline SN-11,191 & 30 & 5 & 2 & 1 & 0 & 0 & $3.1-4.5$ & 3.7 & $15-30$ & \\
\hline \multirow[b]{2}{*}{$\mathrm{SN}-12,325$} & 30 & 5 & 2 & 1 & 0 & 3 & $2.7-4.6$ & 3.5 & 15 & \\
\hline & 60 & 5 & 1 & 3 & 0 & 0 & $4.1-7.6$ & 5.6 & 45 & $\begin{array}{l}1 \text { patient showed white } \\
\text { count of } 3,000-4,000 \\
\text { toward end of course }\end{array}$ \\
\hline \multirow{2}{*}{$\mathrm{SN}-12,352$} & 30 & 5 & 1 & 0 & 0 & 1 & $1.8-4.1$ & 2.9 & 15 & \\
\hline & 60 & 5 & 3 & 3 & 0 & 1 & $1.2-4.5$ & 2.8 & 15 & \\
\hline \multirow{2}{*}{ SN-12,354 } & 30 & 2 & 0 & 0 & 0 & 0 & $2.8-3.1$ & 2.9 & 15 & \\
\hline & 60 & 5 & 1 & 2 & 0 & 3 & $2.7-6.2$ & 4.6 & 30 & \\
\hline \multirow{2}{*}{$\mathrm{SN}-12,451$} & 30 & 3 & 1 & 0 & 0 & 1 & $3.0-5.9$ & 4.1 & $15-30$ & \\
\hline & 60 & 5 & 2 & 1 & 0 & 2 & $3.2-7.2$ & 4.9 & 30 & \\
\hline \multirow[b]{2}{*}{$\mathrm{SN}-13,232$} & 30 & 5 & 0 & 1 & 0 & 2 & $2.5-4.7$ & 3.6 & 15 & \\
\hline & 60 & 5 & 0 & 1 & 1 & 2 & $2.7-4.6$ & 3.0 & 15 & $\begin{array}{l}\text { Pruritic vesicular rash in } \\
2 \text { patients at start of } \\
\text { course, disappeared in } 5 \\
\text { days. Fever of } 101.2 \text { on } \\
8 \text { th day in } 1 \text { patient. }\end{array}$ \\
\hline SN-13,233 & 60 & 5 & 2 & 1 & 2 & 1 & $2.9-6.4$ & 4.8 & 30 & $\begin{array}{l}\text { Fever of } 100.4-100.8 \text { on } \\
8 \text { th and } 9 \text { th days in } 1 \\
\text { patient. }\end{array}$ \\
\hline \multirow{2}{*}{ SN-13,274 } & 30 & 5 & 0 & 4 & 1 & 1 & $2.3-3.0$ & 2.4 & 30 & \\
\hline & 60 & 10 & 5 & 4 & 0 & 8 & $2.1-7.6$ & 4.8 & 30 & \\
\hline
\end{tabular}


TABLE III-Continued

\begin{tabular}{|c|c|c|c|c|c|c|c|c|c|c|}
\hline \multirow[t]{2}{*}{ Drug } & \multirow[t]{2}{*}{$\begin{array}{l}\text { Daily } \\
\text { dose } \\
\text { (base) }\end{array}$} & \multirow[t]{2}{*}{$\begin{array}{l}\text { Number } \\
\text { of } \\
\text { patients }\end{array}$} & \multicolumn{3}{|c|}{$\begin{array}{l}\text { Number of patients } \\
\text { showing commonly } \\
\text { observed symptoms }\end{array}$} & \multirow{2}{*}{$\begin{array}{l}\text { Number } \\
\text { of } \\
\text { patients } \\
\text { showing } \\
\text { leucocy- } \\
\text { tosis of } \\
12,000 \\
\text { or over }\end{array}$} & \multicolumn{2}{|c|}{$\begin{array}{l}\text { Average methemo- } \\
\text { globinemia* on } \\
\text { last } 4 \text { days of } \\
\text { treatment } \\
\text { per cent of total } \\
\text { hemoglobin }\end{array}$} & \multirow{2}{*}{$\begin{array}{c}\text { Approximate } \\
\text { pamaquin } \\
\text { (base) } \\
\text { equivalent } \\
\text { (concurrent } \\
\text { with } \\
\text { quinine) }\end{array}$} & \multirow[t]{2}{*}{ Comment } \\
\hline & & & Mild & $\begin{array}{l}\text { Mod- } \\
\text { erate }\end{array}$ & Severe & & Range & Mean & & \\
\hline \multirow{4}{*}{ SN-13,276 } & $\begin{array}{l}m g m . \\
15\end{array}$ & 5 & 2 & 0 & 0 & 1 & $2.1-2.5$ & 2.3 & $\begin{array}{c}\text { mgm. per day } \\
15\end{array}$ & $\begin{array}{l}1 \text { patient had a macular } \\
\text { erythematous rash. }\end{array}$ \\
\hline & 30 & 10 & 4 & 1 & 0 & 2 & $1.0-4.9$ & 2.8 & 15 & \\
\hline & 45 & 5 & 2 & .1 & 0 & 0 & $2.1-2.8$ & 2.6 & 15 & \\
\hline & 60 & 43 & 11 & 19 & 1 & 8 & $0.8-10.2$ & 3.7 & 30 & $\begin{array}{l}2 \text { individuals had fever; } \\
\text { one of } 102 \text { on } 8 \text { th day and } \\
\text { the other of } 100.6 \text { and } \\
101.4 \text { on the } 5 \text { th and } 6 \text { th } \\
\text { days. }\end{array}$ \\
\hline SN-13,380 & 60 & 5 & 1 & 0 & 3 & 0 & $2.4-4.2$ & 3.1 & 15 & \\
\hline \multirow{2}{*}{ SN-13,429 } & 30 & 5 & 3 & 2 & 0 & 1 & $1.7-3.2$ & 2.5 & 15 & \\
\hline & 60 & 5 & 2 & 1 & 0 & 3 & $2.2-8.3$ & 4.5 & 30 & \\
\hline SN-13,619 & 60 & 5 & 2 & 1 & 0 & 2 & $1.1-1.7$ & 1.5 & 15 & $\begin{array}{l}1 \text { patient complained of } \\
\text { pruritis of palm and soles } \\
\text { during entire course of } \\
\text { therapy. No objective } \\
\text { changes. }\end{array}$ \\
\hline \multirow{2}{*}{ SN-13,694 } & 30 & 5 & 1 & 2 & 0 & 3 & $1.1-1.4$ & 1.3 & 15 & \\
\hline & 60 & 5 & 1 & 0 & 0 & 1 & $0.7-1.6$ & 1.1 & 0 & \\
\hline SN-13,697 & 120 & 5 & 1 & 2 & 0 & 2 & $1.4-2.1$ & 1.5 & 15 & \\
\hline \multirow{4}{*}{ SN-14,011 } & 3.7 & 5 & 1 & 0 & 0 & 2 & $1.3-2.3$ & 1.6 & 0 & \\
\hline & 7.5 & 5 & 1 & 0 & 0 & 1 & $1.2-1.5$ & 1.3 & $\mathbf{0}$ & $\begin{array}{l}\text { Temperature of } 100.4 \text { on } \\
\text { 8th day in one patient. }\end{array}$ \\
\hline & 15 & 5 & 0 & 0 & 0 & 1 & $1.3-2.4$ & 1.5 & 0 & \\
\hline & 30 & 5 & 0 & 0 & 0 & 3 & $2.0-3.1$ & 2.7 & 0 & \\
\hline \multirow{2}{*}{ DR-15,302 } & 30 & 5 & 1 & 1 & 0 & 2 & $3.0-3.9$ & 3.5 & 15 & \\
\hline & 60 & 5 & 0 & 1 & 0 & 1 & $4.1-6.8$ & 5.4 & 30 & \\
\hline
\end{tabular}

* By the method used (17) the mean methemoglobin concentration of 187 normal individuals was 1.8 per cent of the total hemoglobin (standard deviation: 1.0).

approximate daily dosage equivalent of pamaquin (base).

The most common symptom was pain. Frequently after the first two or three days the patients noted epigastric discomfort which seemed to spread into the retrosternal area, and in a few instances to the shoulder, to the back, neck, or inguinal region. The ache or pain was constant with periodic exacerbation, accompanied in most cases by epigastric tenderness. Analgesics were usually withheld in order not to mask the symptoms of toxicity.

In some instances the pain diminished or disappeared after the first three to eight days of drug administration, but in many it persisted throughout treatment, subsiding gradually within the next two or three days. Other symptoms, all transient, occurring with less uniformity in the various groups, included anorexia, nausea and vomiting, headache, weakness, and diarrhea. 


\section{Physical observations}

Except for cyanosis, which was observed when methemoglobinemia exceeded 5 or 6 per cent, few abnormalities were found on physical examination. Slight pallor was sometimes seen, and epigastric tenderness was common.

Six patients had unexplained fever of $100^{\circ}$ to $102^{\circ} \mathrm{F}$. rectally on the fifth to tenth day of medication, the rise most commonly occurring on the eighth day. The fever rapidly subsided in all cases although treatment was not interrupted. Two of the six patients received $\mathrm{SN}-13,276$ at $60 \mathrm{mgm}$. per day; the other four patients were on other drug regimes.

Skin eruptions occurred in three patients. They were mild and transient. Two were vesicular and pruritic, the other was macular and erythematous. All disappeared after treatment was discontinued.

\section{Laboratory observations}

Leucocytes. Leucopenia between 3,000 and 4,000 per cu. $\mathrm{mm}$. was observed in two patients ( $\mathrm{SN}-9,972$ and $\mathrm{SN}-12,325)$. In both, the low counts were recorded at the end of therapy and rapidly returned to normal.

Low-grade leucocytosis was observed in the last three to seven days of the 14-day course of treatment in 66 individuals. The white counts usually rose to 12,000 to 14,000 per cu. mm. but in an occasional subject attained somewhat higher values. The leucocytosis was not associated with the drug fever mentioned above.

Hemoglobin. Except in the case of pentaquine $(\mathrm{SN}-13,276)$ the number of patients on any individual regime was too small for statistical evaluation of change in hemoglobin. When $\mathrm{SN}-13,276$ at $60 \mathrm{mgm}$. of base per day was administered with quinine there occurred a mean fall of 1 gram of hemoglobin (18). If, however, all other regimes are considered together as one group no net decrease in hemoglobin can be demonstrated.

Methemoglobinemia. Methemoglobinemia was produced by all regimes studied except those with SN-191, SN-13,619, SN-13,694, SN-13,697 and lower doses of $\mathrm{SN}-14,011$.

In a given regime the amount of methemoglobinemia varied considerably from individual to individual but not from day to day in the same
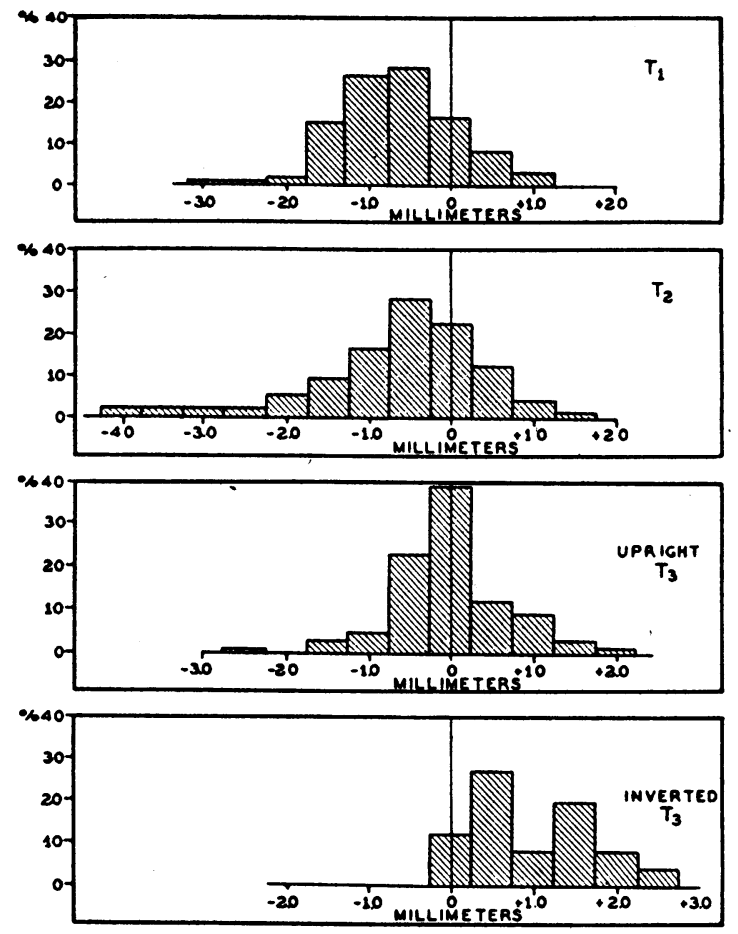

Fig. 4. Histograms of Changes in the Height of T. Waves after the Concurrent Administration of 8-Aminoquinolines and Quinine to 187 Patients during Acute Attacks of VIVAX Malaria

individual. The average of the mean methemoglobinemia during the last four days of treatment for all individuals, and the range of the four-day mean for each individual are shown in Table III.

With the method employed to determine methemoglobin (17) the mean value obtained in the study of 187 normal individuals was 1.8 per cent of the total hemoglobin. (Standard deviation = 1.0.)

Electrocardiographic changes. The electrocardiograms of 187 individuals who had recently completed a 14-day course of an 8-aminoquinoline plus quinine, were studied. Clinical malaria was present only during the first few days of the course of drug administration. The only statistically significant changes noted were diminution of the amplitude of $T_{1}$ and of $T_{2}$, and of $T_{3}$ when initially upright. When $T_{3}$ was initially inverted, it became shallower, or, on occasion, became upright. The distribution and magnitudes of these changes are shown in Figure 4. Previously upright $T$ waves occasionally became inverted in Lead III. This occurred in only one instance in Lead II 
(SN-14,011 + quinine) but never in Lead I. The drug regimes accounting for the greatest $T$ wave changes included the following four drugs (all administered with quinine) : $\mathrm{SN}-9,972, \mathrm{SN}-13,-$ 274, SN-13,276, and SN-14,011. In instances where follow-up electrocardiograms were taken, the $T$-waves rapidly returned to their initial amplitude. We have observed similar changes in drug regimes consisting of 8-aminoquinolines administered without quinine.

\section{DISCUSSION}

Although the toxicity of 8 -aminoquinolines as revealed by animal experiments can serve only as a rough guide to human toxicity, it has been found practical and relatively safe in the study of drugs herein reported to translate the toxicity in the monkey to man, beginning human tests at a dosage estimated to be equivalent to one-sixth the maximum tolerated dose on the basis of comparison with pamaquin. No serious toxicity has been encountered in clinical tests on these regimes. The most prominent symptoms have been abdominal pain, anorexia, nausea and vomiting.

Cyanosis has occurred when methemoglobinemia exceeded 5 or 6 per cent of the total hemoglobin. Mild drug fever, leukocytosis and leukopenia have been observed. The only 8 -aminoquinolines that

TABLE IV

Data on ten 8-aminoquinolines which have "cured" one or more infections presenting a severe therapeutic challenge

\begin{tabular}{|c|c|c|c|c|c|c|c|}
\hline \multirow{2}{*}{$\begin{array}{l}\text { Survey } \\
\text { number }\end{array}$} & \multirow{2}{*}{$\begin{array}{c}\begin{array}{c}\text { General formula of } \\
\text { 8-(w-aminoalkylamino) } \\
\text { quinolines }\end{array} \\
\mathbf{R}^{\prime}-\mathbf{N}-\mathbf{R}^{\prime \prime}\end{array}$} & \multirow{2}{*}{\multicolumn{2}{|c|}{$\sum_{-\left(\mathrm{CH}_{2}\right)_{-}-}^{\mathrm{R}^{\prime}}$}} & \multirow{2}{*}{$\begin{array}{c}\text { Daily } \\
\text { dosage }\end{array}$} & \multirow{2}{*}{$\begin{array}{c}\text { Toxicity: } \\
\text { approximate } \\
\text { pamaquin } \\
\text { daily } \\
\text { dosage } \\
\text { equivalent } \\
\text { (concurrent } \\
\text { with } \\
\text { quinine) }\end{array}$} & \multicolumn{2}{|c|}{$\begin{array}{c}\text { Relapse ratio: } \\
\text { Individuals relapsed } \\
\text { Individuals treated }\end{array}$} \\
\hline & & & & & & $\begin{array}{l}\text { Moderate } \\
\text { infections }\end{array}$ & $\begin{array}{c}\text { Severe* } \\
\text { infection }\end{array}$ \\
\hline & & & & $m g m$. & mgm. per day & & \\
\hline
\end{tabular}

Drugs which have "cured" one infection presenting severe challenge

\begin{tabular}{|c|c|c|c|c|c|c|c|}
\hline SN-12,352 & amino & \multirow{5}{*}{ n-hexyl } & \multirow{4}{*}{ 6-methoxy } & 60 & 15 & $2 / 3$ & $1 / 2$ \\
\hline $\mathrm{SN}-12,451$ & ethylamino & & & 60 & 30 & $0 / 1$ & $3 / 4$ \\
\hline $\mathrm{SN}-13,233$ & $\mathrm{n}$-propylamino & & & 60 & 30 & $0 / 1$ & $3 / 4$ \\
\hline $\mathrm{SN}-12,325$ & methyl isopropylamino & & & 60 & 45 & $2 / 3$ & $1 / 2$ \\
\hline $\mathrm{SN}-13,694$ & diethylamino & & $\begin{array}{l}\text { 5-chloro; } \\
\text { 6-methoxy }\end{array}$ & 60 & 0 & - & $4 / 5$ \\
\hline
\end{tabular}

Drugs which have "cured" more than one infection presenting a severe challenge

\begin{tabular}{|c|c|c|c|c|c|c|c|}
\hline $\mathrm{SN}-13,274$ & \multirow{4}{*}{ isopropylamino } & \multirow{2}{*}{ 1-methylbutyl } & 6-methoxy & 60 & 30 & $0 / 5$ & $1 / 5$ \\
\hline SN-9,972 & & & 5,6-dimethoxy & 15 & 30 & $0 / 2$ & $2 / 10$ \\
\hline $\mathrm{SN}-13,276$ & & \multirow[b]{2}{*}{ n-amyl } & 6-methoxy & 60 & 30 & $1 / 26$ & $3 / 17$ \\
\hline DR-15,302 & & & $\begin{array}{l}\text { 4-methyl; } \\
\text { 6-methoxy }\end{array}$ & 60 & 30 & - & $2 / 5$ \\
\hline SN-13,429 & n-propylamino & 1-methylbutyl & 6-methoxy & 60 & 30 & 一 & $2 / 5$ \\
\hline
\end{tabular}

* Infections were classified as "severe" when the prepatent period or preceding latent interval was less than 15 days or 30 days, respectively. When these periods were longer, the infections were classified as "moderate." 
have not caused significant methemoglobinemia at any dosage level studied are $\mathrm{SN}-191, \mathrm{SN}-13,-$ 619, SN-13,694 and SN-13,697. Three of these ( $\mathrm{SN}-191, \mathrm{SN}-13,619$, and $\mathrm{SN}-13,697)$ have no 6-methoxy radical on the nucleus. The fourth has a chloro substituent in the 5 position as well as a 6-methoxy. The $\mathrm{T}$ wave changes that have been noted are probably non-specific and of dubious significance because they were not attended by other evidence of cardiovascular abnormality and similar changes have been reported with divers other drugs (20 to 25 ).

These studies indicate that the curative properties of pamaquin are not unique to that drug but are shared by several analogues. Nevertheless, the present data do not warrant definite conclusions as to the specific molecular configuration required in a curative drug. Certain tentative inferences, however, suggest themselves.

$\mathrm{SN}-9,972, \mathrm{SN}-13,274, \mathrm{SN}-13,276, \mathrm{SN}-13,429$ and DR-15,302 apparently cured two or more patients (Table IV) when administered concurrently with quinine. They are all secondary amines. The alkyl substituent of the terminal amine nitrogen is isopropyl in four of these drugs and normal propyl in the fifth. All five compounds possess five carbons in the inter-nitrogen aliphatic chain; in three of them it is a secondary amyl and in two it is normal amyl (Table IV). All have a 6-methoxy group on the quinoline nucleus. On an equal weight basis these drugs have greater therapeutic efficacy than pamaquin. SN-13,276 (pentaquine) has been the most extensively studied of these drugs. Investigations on pentaquine are reported in detail elsewhere $(18,19)$.

An additional methoxy group in the 5 position on the quinoline ring appeared to increase the toxicity of the drug. Thus, $\mathrm{SN}-9,972$ is the 5,6dimethoxy homologue of SN-13,274, and SN-12,354 is the dimethoxy homologue of $\mathrm{SN}-12,352$. In each case, the monomethoxy compound producéd less toxicity than the dimethoxy derivative. This is further borne out in the case of $\mathrm{SN}-8,233,4$ which is the 5,6-dimethoxy homologue of pamaquin.

The effectiveness of drugs as curative agents was demonstrated not only by their immediate effect on the relapse ratio but also by their effect on subsequent latent intervals. Drug regimes which produced a high percentage of cures, tended to pro- long the subsequent latent intervals of patients whom they failed to cure. This prolongation was more pronounced with drug regimes which produced a marked lowering of the relapse ratio than with those that decreased the relapse ratio only slightly. The lengthening of the latent interval implies that the disease has been modified and that the relapse rate after subsequent therapy will be lower. The effect of these drugs, therefore, on the clinical course of malaria is greater than is apparent from a study of the immediate relapse ratio alone.

\section{SUM MARY}

1. Eighteen 8-aminoquinolines have been studied for antimalarial activity in human vivax malaria (Chesson strain) and for their clinical toxicity.

2. The relapse rate in this disease is lowered by $\mathrm{SN}-9,972, \mathrm{SN}-13,274, \mathrm{SN}-13,276, \mathrm{SN}-13,429$ and DR-15,302.

3. $\mathrm{SN}-13,276$ (pentaquine) has been the most extensively studied drug of this group. On an equal weight basis it has greater curative activity and lower toxicity than pamaquin (plasmochin).

4. Drug regimes which produced a high percentage of cures tended to prolong the subsequent latent intervals in patients whom they failed to cure.

\section{BIBLIOGRAPHY}

1. Wiselogle, F. Y., editor. A Survey of Antimalarial Drugs, 1941-1945. Edwards Brothers, Inc., Ann Arbor, 1946.

2. Loeb, R. F., Clark, W. M., Coatney, G. R., Coggeshall, L. T., Dieuaide, F. R., Dochez, A. R., Hakansson, E. G., Marshall, E. K., Jr., Marvel, C. S., McCoy, O. R., Sapero, J. J., Sebrell, W. H., Shannon, J. A., and Carden, G. A., Jr., Activity of a new antimalarial agent, chloroquine (SN-7618). Statement approved by the Board for Coordination of Malarial Studies. J. A. M. A., 1946, 30, 1069.

3. Sinton, J. A., and Bird, W., Studies in malaria with special reference to treatment: plasmoquine in treatment of malaria. Indian J. Med. Research, 1928, 16, 159.

4. Sinton, J. A., Smith, S., and Pottinger, D., Studies in malaria, with special reference to treatment. XII. Further researches into treatment of chronic benign malaria with plasmoquine and quinine. Indian J. Med. Research, 1930, 17, 793.

5. Peibenga, P. J., De Malaria-Epidermieen in het geneeskundig gesticht te Franeker en de Gunstige invloed der Chinoplasminebehandeling. Nederl. Tidschrift. voor geneeskunde, 1932, 76² : 1564. 
6. Fourth General Report of the Malaria Commission. Bull. of the Health Organization of the League of Nations, 1937, 6, 895.

7. Feldman, H. R., Packer, H., Murphy, F. D., and Watson, R. B., Pamaquine naphthoate as a prophylactic for malarial infections. Fed. Proc., 1946, $5,244$.

8. Berliner, R. W., Taggart, J. V., Zubrod, C. G., Welch, W. J., Earle, D. P., Jr., and Shannon, J. A., Pamaquin: 1. Curative antimalarial activity in vivax malaria. Fed. Proc., 1946, 5, 165.

9. Craige, B., Jr., Jones, R., Jr., Whorton, C. M., Pullman, T. N., Alving, A. S., and Eichelberger, L., Clinical standardization of Pamaquin (plasmochin) in mosquito-induced vivax malaria (Chesson). A preliminary report. Am. J. Trop. Med., 1947, 27, 309.

10. Office of the Surgeon General, Circular Letter No. 153, The drug treatment of malaria, suppressive and clinical. J. A. M. A., 1943, 123, 205.

11. Alving, A. S., Craige, B., Jr., Pullman, T. N., Whorton, C. M., Jones, R., Jr., and Eichelberger, L., Procedures used at Stateville Penitentiary for the testing of potential antimalarial agents. J. Clin. Invest., 1948, 27, Suppl., 2.

12. Ehrman, F. C., Ellis, J. M., and Young, M. D., Plasmodium vivax, Chesson strain. Science, 1945, 101, 377.

13. Coatney, G. R., Cooper, W. C., Ruhe, D. S., and Young, M. D., Trials of quinacrine, colchicine $(\mathrm{SN}-12,080)$ and quinine against Chesson strain vivax malaria. Am. J. Hyg., to be published.

14. Craige, B., Jr., Alving, A. S., Jones, R., Jr., Whorton, C. M., Pullman, T. N., and Eichelberger, L., The Chesson strain of Plasmodium vivax malaria. II. Relationship between prepatent period, latent period, and relapse rate. J. Infect. Dis., 1947, 80, 228.

15. Brodie, B. B., and Udenfriend, S., The estimation of quinine in human plasma with a note on the estimation of quinidine. J. Pharmacol. \& Exper. Therap., 1943, 78, 154.
16. Brodie, B. B., Udenfriend, S., and Taggart, J. V., Analysis of basic organic compounds in biological tissues: 4. Coupling with diazonium salts. J. Biol. Chem., 1947, 168, 327.

17. Wendel, W. B., Personal communication.

18. Alving, A. S., Craige, B., Jr., Jones, R., Jr., Whorton, C. M., Pullman, T. N., and Eichelberger, L., Pentaquine $(\mathrm{SN}-13,276)$ a therapeutic agent effective in reducing the relapse rate in vivax malaria. J. Clin. Invest., 1948, 27, Suppl., 25.

19. Craige, B., Jr., Jones, R., Jr., Eichelberger, L., Alving, A. S., Pullman, T. N., and Whorton, C. M., The toxicity of large doses of pentaquine (SN$13,276)$ a new antimalarial drug. J. Clin. Invest., 1948, 27, Suppl., 17. .

20. Mainzner, F., and Krause, M., Changes of the electrocardiogram appearing during antimony treatment. Tr. Roy. Soc. Trop. Med. Hyg., 1940, 33, 405.

21. Geiger, A. J., Craige, B., Jr., and Sadusk, J. F., Jr., Arsenotherapy of early syphilis by the intravenous drip method. II. Electrocardiographic abnormalities associated with massive arsenotherapy. Yale J. Biol. Med., 1942, 14, 358.

22. Hartwell, A. S., Burrett, J. B., Graybiel, A., and White, P. D., The effect of exercise and of four commonly used drugs on the normal human electrocardiogram with particular reference to the $T$ wave changes. J. Clin. Invest., 1942, 21, 409.

23. Peters, G. A., and Horton, B. T., Continuous intravenous administration of histamine: Effect on the electrocardiogram and serum potassium. Am. Heart J., 1944, 27, 845.

24. Hardgrove, M., and Smith, E. R., Effect of emetine on the electrocardiogram. Am. Heart J., 1944, 28, 752.

25. Alving, A. S., Eichelberger, L., Craige, B., Jr., Jones, R., Jr., Whorton, C. M., and Pullman, T. N., Studies on the chronic toxicity of chloroquine (SN-7618). J. Clin. Invest., 1948, 27, Suppl., 60. 